\title{
A Rare Infectious Cause of Transverse Myelitis
}

\author{
Gonçalo Cação, Joana Martins, Jose Pedro Rocha Pereira, Ana P. Correia, Joana Damásio
}

Keywords: Transverse myelitis, Enterovirus, Magnetic resonance imaging

doi:10.1017/cjn.2016.308

Can J Neurol Sci. 2017; 44: 110-111

Transverse myelitis has many potential etiologies, among which are demyelinating diseases, connective tissue disorders, infections, paraneoplastic syndromes, and drug/toxin-induced. An infectious etiology is frequently suspected, but the pathogenic agent is rarely identified. Enteroviruses are associated with different neurological syndromes, most commonly in children, ${ }^{1}$ with transverse myelitis being a rare presentation particularly in the adult population. 1,2

We describe a 23-year-old female who presented low back pain associated with lower limb numbness, urinary incontinence, and constipation. There was no viral prodromal illness, namely no respiratory or gastrointestinal symptoms. Two days later, she developed lower limb weakness. Neurological examination disclosed an asymmetric flaccid paraparesis with right predominance, brisk deep tendon reflexes, right Babinski sign, loss of pain sensation below $\mathrm{T} 4$ on the left and $\mathrm{T} 8$ on the right, decreased proprioception, and vibration sense on halluces with loss of ambulation. The patient had no skin rash. Brain magnetic resonance imaging (MRI) was normal, but the spinal cord MRI disclosed a long medullar hyperintensity (Figure 1). Cerebrospinal fluid (CSF) analysis revealed 112 leukocytes (108 monocytes), increased proteins and immunoglobulin $\mathrm{G}$ index, normal glucose, and a positive polymerase chain reaction for enterovirus. Oligoclonal bands were not identified and antiaquaporin 4 antibodies were negative. The remaining CSF and serum studies, including blood count, biochemistry, serology, and immunology, were normal/negative. She was treated with bolus of intravenous methylprednisolone $1000 \mathrm{mg}$ for 5 days followed by intravenous immunoglobulins $0.67 \mathrm{~g} / \mathrm{kg} /$ day for 3 days, and presented immediate improvement. Control MRI, 9 months afterwards, also revealed signs of recovery (Figure 2). With 3 years of follow-up, she presented with minor right lower limb
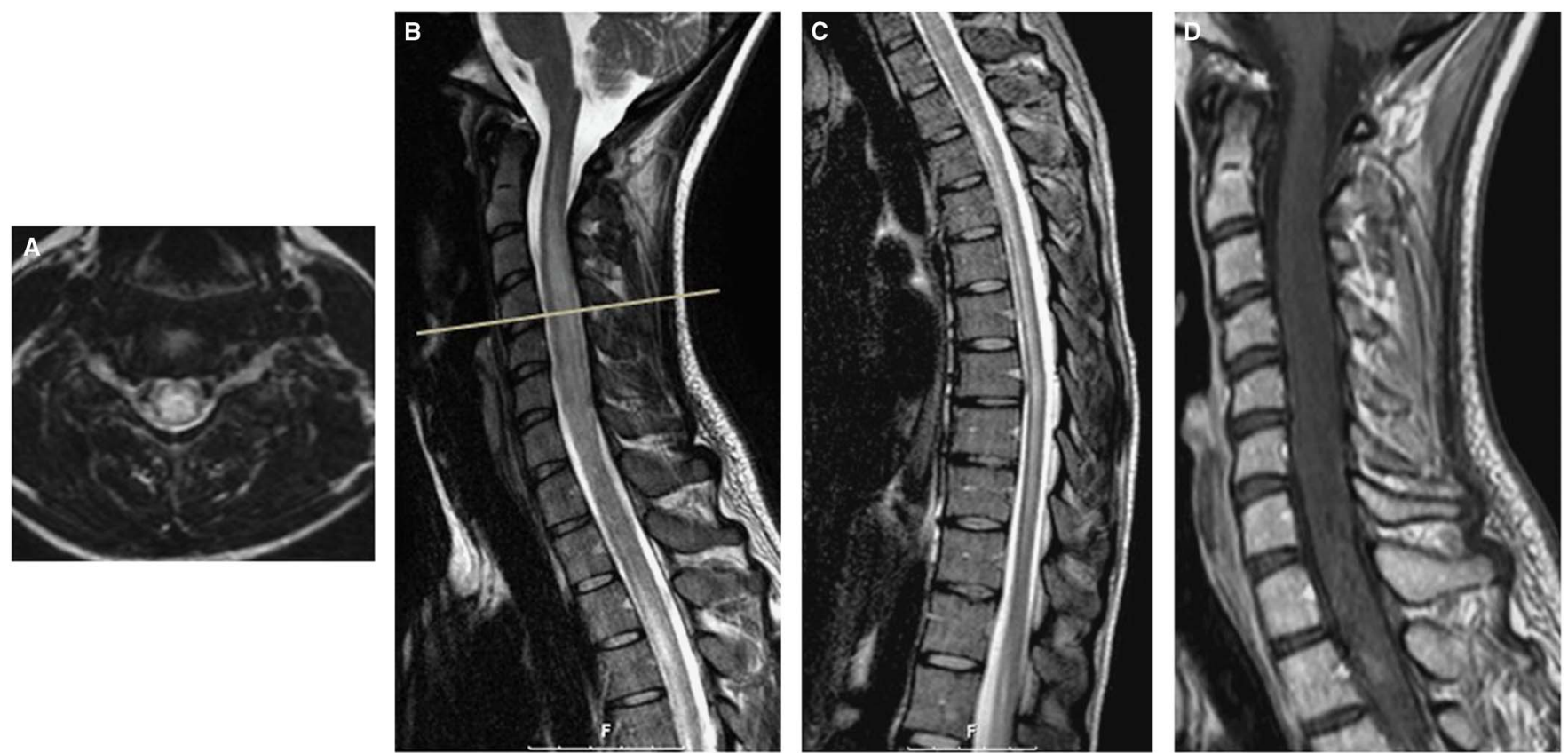

Figure 1: Admission MRI. A, B (axial and sagittal T2): C2-C7 enlargement and transverse medullar hyperintensity affecting more than two-thirds of cord diameter. C (sagittal T2): T3-T11 hyperintensity affecting the anterior cord half. D (sagittal T1): T1-T2 right anterolateral focal contrast enhancement.

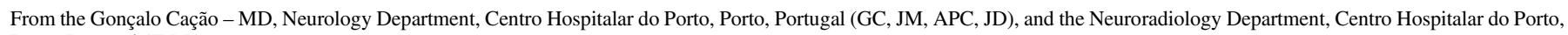
Porto, Portugal (JPRP).

Received May 3, 2016. Final Revisions Submitted July 22, 2016. Date of Acceptance August 9, 2016.

Correspondence to: Joana Damásio, Neurology Department, Centro Hospitalar do Porto, Largo Professor Abel Salazar, $4099-001$ Porto, Portugal.

Email: joanadamasio80@gmail.com 

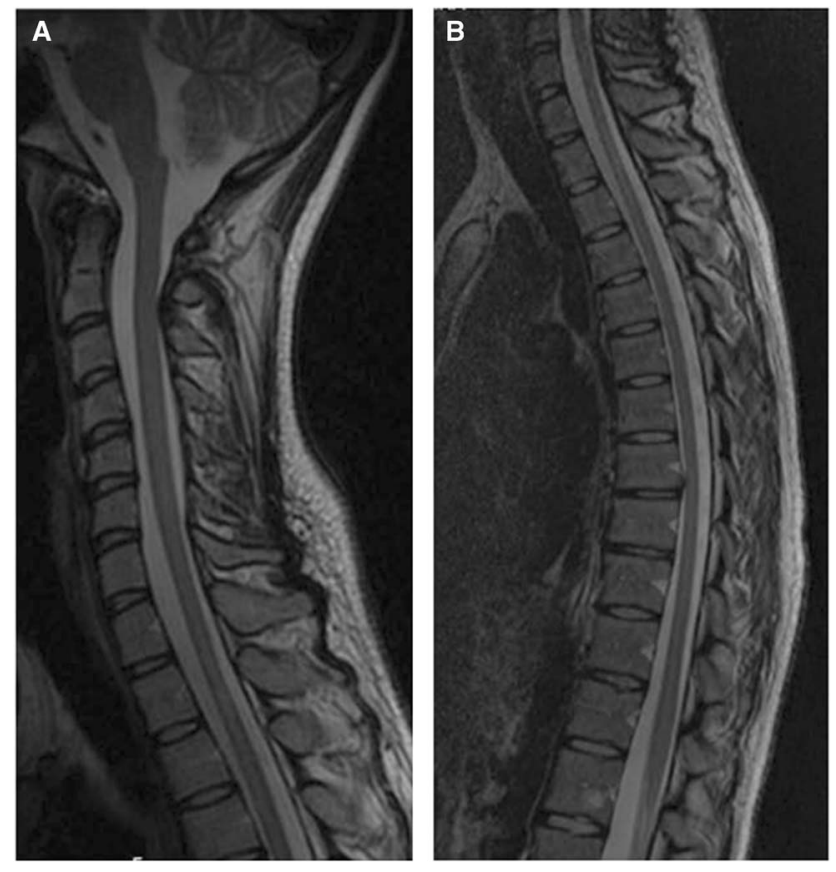

Figure 2: Control MRI 9 months afterwards. A, B (sagittal T2): T1-T3 slight hyperintensity with minor cord atrophy.

weakness with Babinski sign, decreased pain sensation below T8 on the right, and independent ambulation.

We describe a patient with a typical presentation of an acute transverse myelitis (ATM), in whom neither the history, neurological examination, nor spinal MRI pointed to a specific etiology. In ATM, the thoracic cord is the most frequently involved site, with the triad of paraparesis, sensory impairment level, and sphincter dysfunction being the most common clinical signs. Up $40 \%$ of patients with ATM have no findings on MRI, ${ }^{3}$ with the remaining $60 \%$ presenting variable and nonspecific abnormalities. The lesion commonly extends through three to four spinal segments ${ }^{4}$ and occupies more than two-thirds of the cross-sectional area of the cord, ${ }^{4}$ with variable enlargement that is frequently associated to oedema. A viral ATM has no specific imaging abnormalities. In our patient, where a viral prodrome was not present, neither were systemic symptoms, a viral etiology was suspected because of the presence of leukocytes on CSF and later confirmed by polymerase chain reaction.

Enterovirus' tropism for the spinal cord is well-established, although pathophysiological mechanisms remain incompletely understood. $^{2,5}$ Some authors suggest a direct role of the virus causing tissue destruction, whereas others propose an indirect mechanism caused by a cytokine-mediated small-vessel vasculitis. ${ }^{6}$ In the presented case, the major clinical improvement after corticosteroids and immunoglobulins supports an immunomediated mechanism and adds evidence to previous reported cases that also presented a favorable response to corticotherapy. ${ }^{7}$

\section{Statement of Authorship}

Author contributions: The authors contributed equally in the planning, conduct, and reporting of the work described in the article.

\section{Disclosures}

Gonçalo Cação, Joana Martins, Jose Pedro Rocha Pereira, Ana P. Correia, and Joana Damásio do not have anything to disclose.

\section{REFERENCES}

1. Ooi M, Wong S, Lewthwaite $\mathrm{P}$, et al. Clinical features, diagnosis, and management of enterovirus 71. Lancet Neurol. 2010;9: $1097-105$.

2. Cardosa J, Farrar J, Yeng C, et al. A Guide to Clinical Management and Public Health Response for Hand, Foot and Mouth Disease (HFMD). Geneva, Switzerland: World Health Organization Western Pacific Region; 2011.

3. Scotti G, Gerevini S. Diagnosis and differential diagnosis of acute transverse myelopathy. The role of neuroradiological investigations and review of the literature. Neurol Sci. 2001;22(Suppl 2): S69-73.

4. Desanto J, Ross JS. Spine infection/inflammation. Radiol. Clin. North Am. 2011;49:105-27.

5. Teoh HL, Mohammad SS, Britton PN, et al. Clinical characteristics and functional motor outcomes of enterovirus 71 neurological disease in children. JAMA Neurol. 2016;73:300-7.

6. McMinn P, Stratov I, Nagarajan L, et al. Neurological manifestations of enterovirus 71 infection in children during an outbreak of hand, foot, and mouth disease in Western Australia. Clin Infect Dis. 2001;32:236-42.

7. Nolan MA, Craig ME, Lahra MM, et al. Survival after pulmonary edema due to enterovirus 71. Neurology. 2003;60:1651-6. 